\title{
Migratory orientation in a narrow avian hybrid zone
}

\author{
David P L Toews $^{1,2}$, Kira E Delmore ${ }^{1,3}$, Matthew M Osmond ${ }^{1}$, Philip D Taylor ${ }^{4}$, Darren E Irwin ${ }^{\text {Corresp. }}$, $^{1}$ \\ 1 Department of Zoology and Biodiversity Research Centre, University of British Columbia, Vancouver, British Columbia, Canada \\ 2 \\ 3 \\ 3 Current Address: Max Planck Institute for Evolutionary Biology, Plön, Germany \\ 4 Department of Biology, Acadia University, Wolfville, Nova Scotia, Canada \\ Corresponding Author: Darren E Irwin \\ Email address: irwin@zoology.ubc.ca
}

Background. Zones of contact between closely related taxa with divergent migratory routes, termed migratory divides, have been suggested as areas where hybrid offspring may have intermediate and inferior migratory routes, resulting in low fitness of hybrids and thereby promoting speciation. In the Rocky Mountains of Canada there is a narrow hybrid zone between Audubon's and myrtle warblers that is likely maintained by selection against hybrids. Band recoveries and isotopic studies indicate that this hybrid zone broadly corresponds to the location of a possible migratory divide, with Audubon's warblers migrating south-southwest and myrtle warblers migrating southeast. We tested a key prediction of the migratory divide hypothesis, that genetic background would be predictive of migratory orientation among warblers in the center of the hybrid zone.

Methods. We recorded fall migratory orientation of wild-caught migrating warblers in the center of the hybrid zone as measured by video-based monitoring of migratory restlessness in circular orientation chambers. We then tested whether there was a relationship between migratory orientation and genetic background, as measured using a set of species-specific diagnostic genetic markers.

Results. We did not detect a significant association between orientation and genetic background. There was large variation among individuals in orientation direction. Mean orientation was towards the NE, surprising for birds on fall migration, but aligned with the mountain valley in which the study took place.

Conclusions. Only one other study has directly analyzed migratory orientation among naturallyproduced hybrids in a migratory divide. While the other study showed an association between genetic background and orientation, we did not observe such an association in yellow-rumped warblers. We discuss possible reasons, including the possibility of a lack of a strong migratory divide in this hybrid zone and/or methodological limitations that may have prevented accurate measurements of long-distance migratory orientation. 


\section{Migratory orientation in a narrow avian hybrid zone}

4 David P L Toews ${ }^{1,2}$, Kira E Delmore ${ }^{1,3}$, Matthew M Osmond ${ }^{1}$, Philip D Taylor ${ }^{4}$, and Darren E 5 Irwin $^{1}$

$6{ }^{1}$ Department of Zoology and Biodiversity Research Centre, University of British Columbia,

7 Vancouver, British Columbia, Canada

$8{ }^{2}$ Current Address: Fuller Evolutionary Biology Program, Cornell Lab of Ornithology, Ithaca,

9 New York, United States of America

$10{ }^{3}$ Current Address: Max Planck Institute for Evolutionary Biology, Plön, Germany

$11{ }^{4}$ Department of Biology, Acadia University, Wolfville, Nova Scotia, Canada

12

13 Corresponding Author:

14 Darren E. Irwin

15 Department of Zoology and Biodiversity Research Centre,

16 University of British Columbia,

176270 University Blvd.

18 Vancouver, BC V6T 1Z4

19 Canada

20 Phone: $604-882-4357$

21

22

23

24

25

E-mail: irwin@zoology.ubc.ca 


\section{Abstract:}

27

Background. Zones of contact between closely related taxa with divergent migratory routes, termed migratory divides, have been suggested as areas where hybrid offspring may have intermediate and inferior migratory routes, resulting in low fitness of hybrids and thereby promoting speciation. In the Rocky Mountains of Canada there is a narrow hybrid zone between Audubon's and myrtle warblers that is likely maintained by selection against hybrids. Band recoveries and isotopic studies indicate that this hybrid zone broadly corresponds to the location of a possible migratory divide, with Audubon's warblers migrating south-southwest and myrtle warblers migrating southeast. We tested a key prediction of the migratory divide hypothesis, that genetic background would be predictive of migratory orientation among warblers in the center of the hybrid zone.

Methods. We recorded fall migratory orientation of wild-caught migrating warblers in the center of the hybrid zone as measured by video-based monitoring of migratory restlessness in circular orientation chambers. We then tested whether there was a relationship between migratory orientation and genetic background, as measured using a set of species-specific

42 diagnostic genetic markers.

43 Results. We did not detect a significant association between orientation and genetic

44 background. There was large variation among individuals in orientation direction. Mean 45 orientation was towards the NE, surprising for birds on fall migration, but aligned with the 46 mountain valley in which the study took place.

47 Conclusions. Only one other study has directly analyzed migratory orientation among naturally48 produced hybrids in a migratory divide. While the other study showed an association between 
49 genetic background and orientation, we did not observe such an association in yellow-rumped

50 warblers. We discuss possible reasons, including the possibility of a lack of a strong migratory

51 divide in this hybrid zone and/or methodological limitations that may have prevented accurate

52 measurements of long-distance migratory orientation.

53 
54

\section{Introduction:}

Breeding and wintering ranges of many species are separated by thousands of kilometers.

57 Long-distance seasonal migration exhibited by many taxa moving between these disjunct areas is

58 a complex and energetically demanding task that has been studied for decades (Berthold 1996).

59 However, the physiological mechanisms, controls and senses involved in navigation of many

60 taxa are still unclear, as are the contributions of various cues to migratory behavior (Berthold and

61 Terrill 1991; Alerstam 2006). Much of our understanding of migratory directionality and

62 navigation comes from studies of blackcap warblers (Sylvia atricapilla) in Europe. In an

63 influential series of studies, Helbig and colleagues (Helbig 1991; Berthold et al. 1992; Helbig

64 1994; Helbig 1996) reared blackcaps from populations that exhibited different migratory routes.

65 They found that, even in captivity in a common environment, populations recapitulated their

66 natural routes, as assayed by Emlen funnels (Emlen 1970). These studies also found that lab-

67 crossed hybrids between the parental types tended to show intermediate migratory orientations.

68 Few studies have collected similar data in wild populations, primarily due to logistical

69 challenges. Such observations are particularly pertinent to studies of migratory divides, where

70 populations that differ in migratory directionality come into contact and interbreed (e.g., Helbig

71 1991; Bensch et al. 1999, 2009; Irwin and Irwin 2005; Ruegg 2007; Rohwer and Irwin 2011;

72 Ilieva et al. 2012). For example, if hybrids between divergent populations have a mixture of

73 alleles responsible for migration and exhibit an intermediate migratory orientation, as has been

74 observed in lab-raised blackcap warblers (Helbig 1991; Helbig 1994; Helbig 1996), such a novel

75 phenotype may be inferior and represent an important fitness detriment. This is because, in a

76 number of systems, intermediate routes have been suggested to take hybrid individuals over

77 regions that are provide greater challenges in finding food (e.g., deserts) or navigating (e.g., 
78 mountains). If so, inferior routes of hybrids can cause lower survival and/or lower fecundity (if

79 arriving on the breeding grounds in poor condition hampers reproduction), thereby contributing

80 to postmating reproductive isolation and promoting further differentiation and speciation of the

81 two populations (Helbig 1991; Irwin and Irwin 2005).

82 To date, the only study to directly assay the migratory behaviour of wild individuals

83 differing in their genetic constitution across a migratory divide is in a study of Swainson's

84 thrushes (Catharus ustulatus; Delmore et al. 2012; Delmore and Irwin 2014; Delmore et al.

85 2016). In a hybrid zone between coastal and inland forms of Swainson's thrushes in western

86 Canada, migration was studied using both light-level geolocators, to directly track migration

87 routes and wintering locations (in Central and South America), and orientation chambers to

88 measure migratory direction of birds beginning fall migration. Hybrids exhibited greater

89 variability in their routes than parentals did, with some taking routes that were intermediate to

90 parental forms and took them over the arid regions of the southwestern USA. Moreover, among

91 Swainson's thrushes within the hybrid zone, genetic background was predictive of migratory

92 route as well as orientation direction, and one particular genomic region showed a particularly

93 strong association with longitude (i.e., west to east) of migratory route and wintering location

94 (Delmore et al. 2016).

95 Given the prominence of the idea that differences in migratory behavior may cause

96 reproductive isolation within hybrid zones (e.g., Helbig 1991; Bensch et al. 1999, 2009; Bearhop

97 et al. 2005; Irwin and Irwin 2005; Ruegg 2007; Rolshausen et al. 2009, 2013; Liedvogel et al.

98 2011; Rohwer and Irwin 2011; Ilieva et al. 2012), it is important to test whether there is

99 association between genetic background and migratory orientation within a variety of hybrid

100 zones. Here, we provide another direct examination of this question with a large-scale study of 
101 birds sampled on fall migration within a hybrid zone between Audubon's / myrtle warblers

102 (Setaphaga auduboni and S. coronata) in western North America. Within this hybrid zone there

103 are a full range of hybrid genotypes, indicating that multiple generations of hybrids and

104 backcrosses are present and that reproductive isolation is far from complete. However, the

105 narrowness of this hybrid zone and sizeable amounts of linkage disequilibrium in the center of

106 the zone suggest that some form of moderately strong selection maintains it (Brelsford and Irwin

107 2009). Assortative mating and other pre-mating reproductive barriers are unlikely to be strong

108 between these taxa (Brelsford and Irwin 2009; Brelsford et al. 2011; Toews et al. 2014a),

109 implying a potentially sizeable role for post-mating selection against hybrids, possibly based on

110 inferior migratory behaviour. Band recovery and isotopic data from birds outside the hybrid zone

111 suggests the two parental taxa differ in their migratory movements (Figure 1; Toews et al.

112 2014a,b). If migratory traits are genetically controlled and inherited additively — as the studies of

113 blackcaps and Swainson's thrushes suggest—we expected a correlation of orientation and

114 ancestry in the hybrid zone. Specifically, we predicted that individuals more genetically

115 Audubon's-like would orient SSW, individuals more myrtle-like would orient SE, and hybrids

116 would orient intermediate between these two (i.e. south).

117 We used a video-based orientation cage method, initially developed by Fitzgerald and

118 Taylor (2008). These orientation cages are similar in many respects to traditional Emlen funnels

119 (Emlen 1970), but in this case the behaviour of birds was scored using video cameras. As

120 compared to the scratch marks quantified with Emlen funnel experiments, this method has a

121 number of benefits, including: (1) scoring of the videos can be automated, ensuring objectivity,

122 (2) a longer period of observation can be obtained for each individual, (3) specific time periods

123 can be analyzed in isolation and (4) any behavioural changes over the course of the trial can be 
124 quantified. We designed our study to assay the orientation of individuals in the evening of the

125 day they were captured, which previous research suggests is predictive of later orientation (Ilieva

126 et al. 2012). We then genotyped each individual with species diagnostic genetic markers and

127 tested whether there was a correlation between orientation and genetic background.

\section{Methods}

129 Study site and orientation trials

130 Between August $15^{\text {th }}$ and September $12^{\text {th }}$ of 2011 we captured migratory yellow-rumped

131 warblers $(n=181)$ near Kananaskis, Alberta, a site at the center of the Audubon's / myrtle

132 warbler hybrid zone (Brelsford and Irwin 2009). Our sampling was concentrated in two areas:

133 along the southern edge of Barrier Lake $\left(51.0235^{\circ} \mathrm{N}, 115.0608^{\circ} \mathrm{W}\right)$ and within the hamlet of Lac

134 Des $\operatorname{Arc}\left(51.0536^{\circ} \mathrm{N}, 115.1589^{\circ} \mathrm{W}\right.$; Figure 1C). We set up mist-nets before dawn and used

135 passive netting along with song playback (using a tape of a variety of yellow-rumped warblers as

136 well as begging calls from nestlings; previous experience showed that similar recordings could

137 be used to attract all phenotypes in the hybrid zone) to increase our likelihood of catching

138 individuals of our target species. These sites, timing, and capture methodology were chosen to

139 capture birds that were beginning their migratory movements, but it is possible that some of the

140 individuals sampled were close to their breeding territories or natal areas. Immediately after

141 capturing each individual we took morphometric measurements (bill, tarsus, wing and tail length;

142 according to Pyle 1997), photographs, a blood sample (10-40 $\mathrm{L}$ ), and applied a unique

143 aluminum USFWS leg band. Birds were then transported approximately three kilometers from

144 the capture site to the location where the orientation trials were performed $\left(51.0286^{\circ} \mathrm{N}\right.$,

$\left.145115.0242^{\circ} \mathrm{W}\right)$. This site is a large, recently clear-cut field $\left(\sim 400 \mathrm{~m}^{2} ;<10\right.$ years old $)$ near the

146 Kananaskis Biogeoscience Institute and has clear views in all directions. 
148 cages (Figure 2) and given water and small mealworms throughout the day. The cages were a

149 modified design based on Fitzgerald and Taylor (2008), who used similar cages to study

150 orientation in yellow-rumped warblers (Figure 2). The cages were leveled, oriented with a

151 compass, and spaced approximately 3-5 meters apart. The cage frames were made out of pine

152 boards, with the top and bottom of the cages made from composite plywood. The perch was

153 made with a 9" plastic embroidery hoop, held up by four 7/16" dowels, and placed horizontally

154 such that birds could perch on the hoop (Figure 1C). Since research suggests that birds may use

155 magnetic cues for navigation, the wood pieces were joined with non-magnetic brass screws and

156 stainless steel staples were used to affix screen mesh to the top and sides of the cage (Alerstam

157 1993; Muheim et al. 2006). During the day the sides of the cages were covered with a blue,

158 opaque tarp for sun shelter, with part of the tarp rolled up to provide sufficient airflow (Figure

159 2A). Using the same cages to both hold the individuals during the day and run the orientation

160 trials in the evening has a number advantages over traditional use of Emlen funnels (Emlen

161 1970). Most importantly, this likely reduces the stress inherent in holding individuals indoors in

162 artificial environments and moving individuals between holding and assay cages shortly before

163 the trials, as is necessary with funnels (Emlen 1970). We were careful in assessing the welfare of

164 the birds: if individuals did not immediately fly to the perch, showed any signs of stress (e.g.

165 panting), or were not eating properly they were released during the day (less than $15 \%$ of birds

166 captured).

167 Before we started each orientation trial, we removed the food and water dishes and closed

168 each tarp around the sides of each cages, allowing each individual to see only out of the top of

169 the cage, which had a full view of the sky. Through a hole in the bottom of each cage we 
170 attached a D-Link Wireless Network Camera (DCS-932L) pointing directly up, with the top of

171 the camera oriented northwards. Unlike cameras that point down (e.g. Muheim et al. 2014), these

172 upward-facing cameras do not obstruct a bird's view of the sky. The cameras we used have an

173 infrared LED light for illumination during low light conditions. This light was applied

174 consistently across all of the trials, and we are aware of no evidence that birds can see infrared

175 light. The cameras were set such that the video image was recorded with the right side of the

176 video representing the west side of the cage and the left side of the video representing the east

177 side of the cage. We recorded $320 \mathrm{p}$ x 240p 30 frame-per-second video from each cage

178 simultaneously using the D-ViewCam software on a PC laptop via a D-Link router. We began

179 recording approximately one hour before sunset and ran the trials until approximately 30 minutes

180 past sunset. Each evening we ran the trials until the last individual stopped moving, after which

181 we released all of the individuals. We tested each bird once and recorded the behaviour of 1 to

18210 individuals each evening, averaging 6 birds per evening, with a total of 124 individuals for

183 which we collected complete orientation data. All animal care and experimentation was

184 conducted according to the University of British Columbia protocol Nos. A11-0054 (Project

185 title: Orientation in migratory songbirds) and A09-0131 (Project title: Geographic variation in

186 birds of western Canada). Field permits were provided by the Canadian Wildlife Service, Prairie

187 and Northern Region office (AB Scientific Permit 11-AB-SC023) and Alberta Parks (11-107).

188 Video analysis

189 From the D-ViewCam software we exported each video in ".asf" format, noting the start

190 and end time of each trial. We analyzed the video data with radR, an open source platform

191 developed for acquiring and analyzing radar data (Taylor et al. 2010) that was more recently

192 adapted to analyze video files. In brief, radR uses contrast to score individual pixels, and then 
193 uses movement, area and intensity to define objects. We sampled the videos at three frames per

194 second. For each detected object we extracted the X and Y coordinates of its centroid, as

195 weighted by the area of the object. Given that the light conditions change over the evening, we

196 used three groups of parameters to accurately and consistently identify the bird as the primary

197 object relative to the background (see supplementary material for parameters used). We defined

198 the center of the circular perch as the center of our analytical coordinate system and created an

199 exclusion zone within the circular perch, such that the program would identify the bird only in

200 the region from the perch outwards (Figure 3). This makes our results more comparable to

201 previous funnel studies, which only recorded jumps where the individual contacts the side of the

202 funnel (Fitzgerald and Taylor 2008). From this data radR generates a list of time-stamped X and

203 Y coordinates for points where it identified an object (see supplementary material for an example

204 of data extracted for a single individual).

205 Using R 3.0.3 (R-core Development Team 2013) we applied a number of additional

206 filters, primarily to remove noise (i.e. objects that were not the bird) and remove times when the

207 bird was not moving (i.e. sitting on the perch). To remove noise, we first removed all data where

208 there were three or more objects identified at a single time-stamp, as three or more objects were

209 invariably an artifact of background noise. Sometimes radR identified the bird as two separate

210 objects, primarily when the individual was above one of the four perch dowels, such that parts of

211 the bird stuck out on either side of the dowel. For time-stamps with two objects, we averaged the

$212 \mathrm{XY}$ values that were less than 500 pixels apart to include only those times that an individual was

213 above the dowels (i.e. the dowels are slightly less than 500 pixels wide in the video image) and

214 visually inspected the data to confirm this was accurate. To ignore points where the bird was

215 sitting motionless on the perch we removed consecutive time points that had a lower XY 
216 distance than a predetermined threshold, which we calculated by studying videos of individuals

217 sitting quietly on the perch (50 pixels for the current analysis). Each of the points that passed

218 these filters were then transformed into an angle relative to North $\left(\mathrm{N}=0^{\circ}, \mathrm{E}=90^{\circ}, \mathrm{S}=180^{\circ}\right.$ and

$219 \mathrm{~W}=270^{\circ}$ ) and given a timestamp (in seconds) relative to the time of sunset for that evening.

220 From these data we estimated three behavioural traits for each individual: mean

221 orientation, rho and activity, defined as follows. We calculated mean orientation using the

222 "circular statistics" package in R (Agostinelli and Lund 2007). We used the mean of angles

223 observed over the entire observational period (restricting the time range to only those times when

224 the birds were more active did not meaningfully change the results). We used the same $\mathrm{R}$

225 package to estimate rho, an estimate of angular variance that varies between 0 and 1 (i.e. a

226 measure of the concentration of points, with a value of 1 being perfectly concentrated). Finally,

227 we used the total number of time points at which an object (i.e., a moving bird) was detected by

228 radR over the trial as the total activity for each individual.

229 To assess robustness of estimating the mean orientation for each individual using the data

230 generated from radR, we chose five orientation videos at random and scored them by eye, blind

231 to the output from radR. For this we visually estimated the angle of the bird (if present in the

232 frame) every 30 seconds, over the entire video, and also recorded whether the bird was on the

233 perch or in mid-flight. We then calculated the difference in angle between this estimate and that

234 obtained from radR for the full dataset and also from only those points where the bird was

235 observed to be in flight. Our data filtering appeared to be effective as our data from a random

236 selection of videos analyzed by eye (for 5 birds) was consistent with the output from radR. When

237 we compared all of the points observed by eye, including those where the individual was sitting

238 on the perch, the resultant mean angles were within $+/-22^{\circ}$ relative to the output from radR after 
239 filtering. If we included only those points where the individual was in flight (by excluding those

240 times we observed by eye for which the individual was on the perch), which our filters within

$241 \mathrm{radR}$ were designed to remove, the resultant mean angles were within $+/-11^{\circ}$ of the radR output.

242

243 Molecular analysis

244 Blood samples were taken using a small needle and capillary tube from the brachial vein,

245 stored in Queen's lysis buffer (Seutin et al. 1991), and left at ambient temperature until returned

246 to the laboratory. DNA was extracted using a standard phenol-chloroform procedure and

247 resuspended with $50-200 \mu \mathrm{L}$ of buffer (depending on the size of the pellet) containing $10 \mathrm{mM}$

248 Tris- $\mathrm{HCl}$ and $1 \mathrm{mM}$ EDTA, at $\mathrm{pH} 8.0$, and stored at $4^{\circ} \mathrm{C}$. We sexed individuals molecularly

249 using the procedure described in Fridolfsson and Ellegren (1999). We genotyped individuals at

250 three molecular markers. The full PCR and genotyping protocol for two of the three nuclear

251 markers (CHDIZ and numt-Dcol) was presented in a previous publication (Brelsford and Irwin

252 2009). For the third nuclear marker, an 850 base pair fragment of $R I O K 2$, we used the forward

253 primer 5'-ATGGGTGTTGGCAAAGAATC-3', the reverse primer 5'-

254 GCTCCTCTTCRTTWGCAACA-3', and a PCR annealing temperature of $60^{\circ} \mathrm{C}$. The enzyme

$255 X m n I$ cuts an allele common in Audubon's warblers, but leaves intact an allele common in

256 myrtle warblers. To generate a genetic hybrid index we scored a zero for each Audubon's allele

257 and a one for each myrtle allele for the three markers and divided this by the total number of

258 alleles ( 6 for males, 4 for females) resulting in an index that ranges between 0 (all Audubon's

259 alleles) to 1 (all myrtle alleles). 
260

261

262

263

264

265

266

267

268

269

270

271

272

273

274

275

276

\section{Results}

278

279

280

281

282

\section{Combining genotype and migratory behaviour}

To test whether orientation is associated with genetic background, we used two statistical approaches. First, we used a circular linear model to test whether orientation varied linearly with hybrid index (Agostinelli and Lund 2007). Second, we used a circular ANOVA to test whether mean orientation angle differed in any way (not necessarily linearly) among these five genetic groups: those with all Audubon's alleles (h-index = 0; Group A), those with mostly Audubon's alleles $(0<\mathrm{h}$-index $<0.5$; Group $\mathrm{AH})$, those with mixed genotypes (h-index $=0.5$; Group $\mathrm{H}$ ), those with mostly myrtle alleles $(0.5<$ h-index $<1$; Group $\mathrm{MH})$, and those with all myrtle alleles (h-index $=1$; Group M). While the linear model would assume that each hybrid class as an intermediate migratory orientation compared to the two classes on either side of it, the ANOVA does not make that assumption, allowing situations in which hybrid classes might have more extreme orientations than either parental group.

To test whether there was a significant mean orientation of all of individuals considered together, regardless of their genetic background, we used a Rayleigh test. This is a procedure to test the null hypothesis that the orientation angles are distributed randomly, with the alternative being that the distribution is clumped in certain direction(s). The test statistic is $r$, or rho, and is the magnitude of the mean vector (Fitzgerald and Taylor 2008).

\section{Molecular data}

We obtained genotypes from 166 of the 181 yellow-rumped warblers captured at the beginning of fall migration through the Kananaskis area, including 123 individuals with orientation data. Based on the three genetic markers, our data set consisted of individuals with a broad range of genetic backgrounds, spanning from Audubon's warblers $(\mathrm{h}$-index $=0)$ through a 
283 broad array of hybrid genotypes to myrtle warblers $(\mathrm{h}$-index $=1)$, consistent with the expectation

284 based on prior research of a broad mixture of hybrid classes (F1's, F2's, backcrosses, etc.) and

285 pure-type individuals (Brelsford and Irwin 2009). The daily composite allele frequencies varied

286 relatively little over the study period: the daily proportion of myrtle alleles was usually between

2870.3 to 0.6 , with an average over the study period of 0.4 (Figure 4A). Our molecular sexing of

288 individuals indicated that we captured an excess of male birds with $73 \%$ of all of the individuals

289 identified as males (Figure 4B). This was likely due to our use of song playback during mist

290 netting.

\section{Orientation trials}

292 In the orientation trials, initial observations of yellow-rumped warblers in the orientation

293 chambers indicated that their activity begins to increase approximately one hour before sunset.

294 During this time of increased activity their behavior also changed qualitatively, from primarily

295 sitting on the perch and/or flying occasionally to and from the bottom of the cage, to performing

296 more short flights from the perch to the top of the cage, consistent with zugunruhe (i.e. migratory

297 restlessness; Emlen 1970). The activity of the birds increased to a peak at around 20-30 minutes

298 before sunset (Figure 5), and then gradually declined. Following sunset their activity sharply

299 declined, such that we recorded virtually no movements after 40 minutes post-sunset. At the end

300 of most evenings individuals usually stopped moving within 5-10 minutes of each other with

301 remarkable consistency (the steep decline to the right of Figure 5).

302 Of the 123 individuals where we had genotype and orientation data, we found that 96

303 birds showed strong evidence of orientation behavior (i.e., within-individual $r>0.1$; total

304 activity $>500$ ). We used all 123 individuals in analyses; analyses using only those individuals

305 with strong orientation did not qualitatively change results. Of the five genetic groups, our 
306 sample contained $n=6$ for Group A (i.e., genetically Audubon's), $n=45$ for Group AH, $n=41$

307 for Group H, $n=22$ for Group MH and $n=9$ for Group M (i.e., genetically Myrtle). We did not

308 detect a significant difference in mean orientation between the genetic groups $(F=1.23, P=$

309 0.09) and there was no significant linear relationship between mean orientation and genetic

310 hybrid index $(t=0.779, P=0.22)$. Hence we could not reject the null hypothesis of no

311 association between genetic background and orientation.

312 While there was considerable variability in orientation among individuals, there was a

313 significant mean orientation towards $25^{\circ}$ or NNE (Figure $6 \mathrm{~A} ; n=123$, among-individual $r=$

$3140.320, P<0.01$ ), with a $95 \%$ confidence interval between $5^{\circ}$ and $46^{\circ}$. Separating the birds by

315 their genetic hybrid index, each group had mean orientations similar to N or NE, although only

316 Groups $\mathrm{M}(\mathrm{h}$-index $=1)$ and $\mathrm{H}(\mathrm{h}$-index $=0.5)$ showed evidence of significant mean orientations

317 compared to the null of an even distribution of orientation angles: Group A, $38^{\circ}(n=6, r=$

318 0.634, $P=0.09)$; Group AH, $3^{\circ}(n=45, r=0.230, P=0.09)$; Group H, $54^{\circ}(n=41, r=0.373, P$

$319<0.01)$; Group MH, $359^{\circ}(n=22, r=0.321, P=0.10)$; and Group M, $15^{\circ}(n=9, r=0.733, P<$

320 0.01).

\section{Discussion}

322

Here we have provided the second study of potential association of genetic ancestry and

323 migratory orientation in an avian hybrid zone. Our use of video-based orientation trials and

324 automated video analysis enabled us to sample a large number of individuals during fall

325 migration while also gathering high resolution and orientation chamber movement data for each

326 bird, a benefit over previous Emlen funnel methods. Despite these methodological advantages

327 and prior evidence for a possible migratory divide across the Audubon's / myrtle warbler hybrid

328 zone (Toews et al. 2014a), we did not find an association between genetic background and 
329 orientation. We discuss below the possibilities that this result was due to (1) lack of any true

330 association between genetic background and migratory route within this hybrid zone, (2) short-

331 term orientation during the time of the study not being indicative of long-distance migration

332 orientation; or (3) methodological limitations that hindered the detection a true association.

333 First, the prior evidence for a migratory divide across the yellow-rumped warbler hybrid

334 zone is mixed, raising the possibility that our results are due to no actual association of genetic

335 ancestry and migratory differences in the center of the zone. Banding data and wintering range

336 maps of Audubon's and myrtle warblers indicate that Audubon's warblers largely winter in the

337 southwestern USA, whereas myrtle warblers mostly winter in the southeastern USA (Toews et

338 al. 2014a). However, sizeable numbers of myrtle warblers winter in the southwest (Hunt and

339 Flaspohler 1998). It has been postulated that most of these belong to a distinct form of myrtle

340 warbler (the subspecies hooveri) breeding in Alaska, the Yukon, and northern British Columbia

341 (McGregor 1899; Toews 2017). However, it is also possible that some myrtle warblers from

342 Alberta also migrate to the southwestern USA, and that the current hybrid zone between

343 Audubon's and myrtle warblers in western Alberta does not correspond to a migratory divide.

344 Isotopic data (Toews et al. 2014a) is mostly supportive of the hybrid zone coinciding with a

345 migratory divide, because sites just outside of the hybrid zone differed in their hydrogen isotopic

346 signatures in a way consistent with the expected difference between southwestern and

347 southeastern USA, and birds within the hybrid zone showed a broader mixture of signatures.

348 Taking this evidence together, it is likely that there is at least some sort of transition in average

349 migratory orientation between Audubon warblers in central British Columbia and myrtle

350 warblers in central Alberta, but it is possible that that the transition zone is very broad, with birds 
351 in the center of the hybrid zone having no or only a weakly detectable relationship between

352 ancestry and migratory orientation.

353 Second, it is possible that the behavior that we observed in the chambers is indicative

354 only of short-term and short-distance orientation rather than longer-term and longer-distance

355 migratory orientation. Among individuals we found a lot of scatter in directional tendencies, with

356 a mean migratory orientation that was surprisingly towards the NNE $\left(26^{\circ}\right)$, odd for birds on fall

357 migration, which is expected to be generally southward. A possible explanation is that

358 individuals may be moving northeast, out of the valleys in the Rocky Mountains, to later turn

359 south. The orientation of the valley near the capture location and orientation experiment area is

360 approximately $23^{\circ}$ (NNE; estimated using Google Earth $\left.{ }^{\mathrm{TM}}\right)$, very close to the observed

361 orientation of the birds when grouped together $\left(26^{\circ} \mathrm{NNE}\right)$. Our sample likely included many

362 hatch-year birds, raising the possibility that the northward orientation of some birds may be

363 indicative of regional-scale post-fledging movements (such as those made by blackpoll warblers;

364 Brown and Taylor 2015), the incidence and function of which are still unknown. While previous

365 orientation studies have tested the effect of ecological barriers on migratory behaviour (i.e. water

366 bodies; Sandberg and Moore 1996; Ilieva et al. 2012), this is one of the first studies to assay

367 orientation in and around mountainous areas. Using high-resolution radar technology, Williams

368 et al. (2001) found evidence that nocturnal migrants responded to local topological features by

369 changing their orientation during fall migration, especially those birds migrating below $300 \mathrm{~m}$, as

370 is assumed with yellow-rumped warblers. Given the type of data these methods collect, however,

371 it is challenging to assign these types of observations to specific species or even species groups

372 (Williams et al. 2001). For the warblers in our study, individuals might have a memory of the

373 axis of the valley at the time of capture, and then orientation in that direction. We recommend 
374 that future orientation studies around mountainous areas should consider including additional

375 orientation localities in valleys of varying orientations. It would also be useful to assay

376 individuals each evening over a longer period (i.e. 1-2 weeks) to test whether this orientation is

377 maintained or dissipates with time. This could provide a robust test of the role of topological

378 features in influencing migratory movements.

379 Finally, it is possible that the observed chamber orientations are not representative of

380 movements the birds would make if they were outside of the chambers. All experiments using

381 orientation chambers with captive birds run the risk that migratory movements in specific

382 systems may not be well represented by cage movements, although it is remarkable how often

383 there is a strong association (e.g., Helbig 1991, 1996; Alerstam 1993; Van Doren et al. 2017).

384 The movements and pattern of activity of the warblers in our study were qualitatively similar to

385 previous descriptions of zugunruhe, suggesting that the yellow-rumps in our sample were

386 expressing behaviours consistent with migration. But given the unexpected mean orientation

387 toward the northeast, the lack of any relationship with genetic background, and the reasonably

388 short duration of the movement behavior each evening, we acknowledge that it is plausible that

389 orientation cage behaviors in our study are not indicative of orientation in free-moving birds.

390 Given this concern, we suggest that tracking studies using direct tracking technology such as

391 radio towers and miniaturized geolocator tags may be more useful for studying large-scale

392 migratory orientation (Taylor et al. 2011; Delmore et al. 2012; Veen 2013; Delmore and Irwin 393 2014).

394 In conclusion, we used molecular genetic methods and a video-based orientation assay

395 that provided objective, high resolution temporal data for many individuals in a semi-natural

396 setting. Our results show no significant association between genetic ancestry and chamber 
397 orientation of migrating yellow-rumped warblers within a hybrid zone that was postulated to

398 correspond to a migratory divide. These results provide an interesting contrast to Swainson's

399 thrushes, in which genetic ancestry within a hybrid zone is predictive of migratory orientation as

400 measured both by geolocators and orientation chambers (Delmore et al. 2016). We suggest that,

401 with future modifications, these methods could provide a powerful tool for understanding

402 migratory orientation in many species. In particular, when replicated in multiple locations, one

403 could use these orientation assays to examine the effect of local topographical features. Or, if

404 assayed orientations were verified by geolocators, one could identify potentially maladaptive

405 migratory tendencies in hybrid individuals. We hope these methods will contribute to the

406 understanding of migratory divides, and of migration more generally.

407

408

409 Acknowledgments:

410 We thank Stephanie Cavaghan with assistance in the field. The Kananaskis Biogeoscience

411 Institute graciously provided accommodation and logistical support. We thank John Brzustowski

412 for assistance with implementation of the radR analysis. Permits and land access was provided

413 by the Canadian Wildlife Service, Alberta Fish and Wildlife Service, Alberta Parks and

414 Recreation and the Alberta Sustainable Resource Development Land and Forest Division. 
416 Literature Cited:

417

418 Agostinelli C, Lund U. 2013. R package 'circular': Circular Statistics (version 0.4-7). https://rforge.r-project.org/projects/circular/

Alerstam T. 1993. Bird Migration. Cambridge University Press.

421

422

423

424

425

426

427

428

429

430

431

432

433

434

435

436

437

Alerstam T. 2006. Conflicting evidence about long-distance animal navigation. Science 313:791-794.

Bearhop S, Fiedler W, Furness RW, Votier SC, Waldron S, Newton J, Bowen GJ, Berthold P, Farnsworth K. 2005. Assortative mating as a mechanism for rapid evolution of a migratory divide. Science 310:502-504.

Bensch S, Andersson T, Åkesson S. 1999. Morphological and molecular variation across a migratory divide in Willow Warblers, Phylloscopus trochilus. Evolution 53:1925-1935.

Bensch S, Gran M, Müller N, Gay L, Åkesson S. 2009. Genetic, morphological, and feather isotope variation of migratory willow warblers show gradual divergence in a ring. Molecular Ecology 18:3087-3096.

Berthold P. 1996. Control of migration. Chapman \& Hall, London.

Berthold P, Helbig A J, Mohr G, Querner U. 1992. Rapid microevolution of migratory behaviour in a wild bird species. Nature 360:668-670.

Berthold P, Terrill SB. 1991. Recent advances in studies of bird migration. Annual Review of Ecology and Systematics 22:357-378.

Brelsford A, Irwin DE. 2009. Incipient speciation despite little assortative mating: the yellowrumped warbler hybrid zone. Evolution 63:3050-3060. 
438 Brelsford A, Milá B, Irwin DE. 2011. Hybrid origin of Audubon's warbler. Molecular Ecology

439 20:2380-2389.

Brown JM, Taylor PD. 2015. Adult and hatch-year blackpoll warblers exhibit radically different regional-scale movements during post-fledging dispersal. Biology Letters 11:20150593.

443

444

445

446

447

448

449

450

451

452

453

454

455

456

457

458

459

Delmore KE, Fox JW, Irwin DE. 2012. Dramatic intraspecific differences in migratory routes, stopover sites and wintering areas, revealed using light-level geolocators. Proceedings of the Royal Society B: Biological Sciences 279:4582-4589.

Delmore KE, Irwin DE. 2014. Hybrid songbirds employ intermediate routes in a migratory divide. Ecology Letters 17:1211-1218.

Delmore KE, Toews DPL, Germain RR, Owens GL, Irwin DE. 2016. The genetics of seasonal migration and plumage color. Current Biology 26: 2167-2173.

Emlen ST. 1970. Celestial rotation: its importance in the development of migratory orientation. Science 170:1198-1201.

Fitzgerald TM, Taylor PD. 2008. Migratory orientation of juvenile yellow-rumped warblers (Dendroica coronata) following stopover: sources of variation and the importance of geographic origins. Behavioral Ecology and Sociobiology 62:1499-1508.

Fridolfsson A, Ellegren H. 1999. A simple and universal method for molecular sexing of nonratite birds. Journal of Avian Biology 30:116-121.

Helbig A. 1991. Inheritance of migratory direction in a bird species: a cross-breeding experiment with SE- and SW-migrating blackcaps (Sylvia atricapilla). Behavioral Ecology and Sociobiology 28:9-12. 
460 Helbig A. 1994. Genetic basis and evolutionary change of migratory directions in a European 461 passerine migrant Sylvia atricapilla. Ostrich 65:151-159.

462

463

464

465

466

467

468

469

470

471

472

473

474

475

476

477

478

479

Helbig A. 1996. Genetic basis, mode of inheritance and evolutionary changes of migratory directions in Palaearctic warblers (Aves: Sylviidae). Journal of Experimental Biology 199:49-55.

Hunt PD, Flaspohler DJ. 1998. Yellow-rumped warbler (Setophaga coronata). In: Poole A, Gills F, eds. The birds of North America, No. 376. Ithaca: Cornell Lab of Ornithology.

Ilieva M, Toews DPL, Bensch S, Sjoholm C, Åkesson S. 2012. Autumn migratory orientation and displacement responses of two willow warbler subspecies (Phylloscopus trochilus trochilus and P. t. acredula) in South Sweden. Behavioural Processes 91:253-261.

Irwin DE, Irwin JH. 2005. Siberian migratory divides: the role of seasonal migration in speciation. In: Greenberg R, Marra PP, eds. Birds of two worlds: the ecology and evolution of migration. Baltimore, Maryland: Johns Hopkins University Press, 27-40.

Irwin DE, Irwin JH, Smith T. 2011. Genetic variation and seasonal migratory connectivity in Wilson's warblers (Wilsonia pusilla): species-level differences in nuclear DNA between western and eastern populations. Molecular Ecology 20:3102-3115.

Liedvogel M, Åkesson S, Bensch S. 2011. The genetics of migration on the move. Trends in Ecology and Evolution 26:561-569.

McGregor, RC. 1899. The Myrtle Warbler in California and description of a new race. Condor $1: 31-33$. 
480 Muheim R, Moore FR, Phillips JB. 2006. Calibration of magnetic and celestial compass cues

481

482

483

484

485

486

487

488

489

490

491

492

493

494

495

496

497

498

499

500

501

in migratory birds: a review of cue-conflict experiments. Journal of Experimental Biology

209:2-17.

Muheim R, Henshaw I, Sjöberg S, Deutschlander ME. 2014. BirdOriTrack: a new videotracking program for orientation research with migratory birds. Journal of Field Ornithology 85:91-105.

Paxton KL, Yau M, Moore FR, Irwin DE. 2013. Differential migratory timing of western populations of Wilson's Warbler (Cardellina pusilla) revealed by mitochondrial DNA and stable isotopes. The Auk 130:689-698.

R Development Core Team. 2013. R: A language and environment for statistical computing. Vienna, Austria: R Foundation for Statistical Computing.

Rohwer S, Irwin DE. 2011. Molt, orientation, and avian speciation. The Auk 128:419-425.

Rolshausen G, Segelbacher G, Hobson KA, Schaefer HM. 2009. Contemporary evolution of reproductive isolation and phenotypic divergence in sympatry along a migratory divide. Current Biology 19:2097-2101.

Rolshausen G, Segelbacher G, Hermes C, Hobson KA, Schaefer HM. 2013. Individual differences in migratory behavior shape population genetic structure and microhabitat choice in sympatric blackcaps (Sylvia atricapilla). Ecology and Evolution 3:4278-4289.

Ruegg K. 2007. Genetic, morphological, and ecological characterization of a hybrid zone that spans a migratory divide. Evolution 62:452-466.

Sandberg R, Moore FR. 1996. Fat stores and arrival on the breeding grounds: reproductive consequences for passerine migrants. Oikos 77:577-581. 
502 Seutin G, White BN, Boag PT. 1991. Preservation of avian blood and tissue samples for DNA 503 analyses. Canadian Journal of Zoology 69:82-90.

504 Sullivan KA. 1989. Predation and starvation: age-specific mortality in juvenile juncos (Junco 505 phaenotus). The Journal of Animal Ecology 58:275-286.

506

507

508

509

510

511

Taylor PD, Brzustowski JM, Matkovich C, Peckford ML, Wilson D. 2010. radR: an opensource platform for acquiring and analysing data on biological targets observed by surveillance radar. BMC Ecology 10:22.

Taylor PD, Mackenzie SA, Thurber BG, Calvert AM, Mills AM, McGuire LP, Guglielmo CG. 2011. Landscape movements of migratory birds and bats reveal an expanded scale of stopover. PloS ONE 6:e27054.

Toews DPL, Brelsford A, Irwin DE. 2014a. Isotopic variation across the Audubon's and myrtle hybrid zone. Journal of Evolutionary Biology 27:1179-1191.

Toews DPL, Mandic M, Richards JG, Irwin DE. 2014b. Migration, mitochondria and the yellow-rumped warbler. Evolution 68:241-255.

Toews DPL. 2017. Habitat suitability and the constraints of migration in New World Warblers. Journal of Avian Biology, in press.

Van Doren BM, Liedvogel M, Helm B. 2017. Programmed and flexible: long-term Zugunruhe data highlight the many axes of variation in avian migratory behavior. Journal of Avian Biology 48:155-172.

Veen T. 2013. Unraveling migratory connections: the next level. Molecular Ecology 22:41444146. 
523 Williams TC, Williams JM, Williams PG, Stokstad P. 2001. Bird migration through a

524 mountain pass studied with high resolution radar, ceilometers, and census. The Auk

$525 \quad$ 118:389-403.

526

527 Figure Captions:

528 Figure 1 Distribution and band recoveries of Audubon's and myrtle warblers, and location of

529 study site. (A) Banding data obtained from Brewer et al. (2006) and the Canadian Bird Banding

530 Office (2013). Note that there is a distinct subspecies of Audubon's warbler, the black-fronted

531 warbler, that occurs in Mexico and is not distinguished on this map. (B) Sites of capture for

532 migratory yellow-rumped warblers with $(\mathbf{C})$ the site of the orientation assays. Map data: Google,

533 DigitalGlobe.

534 Figure 2 Holding and orientation cages as modified from Fitzgerald and Taylor (2008). (A)

535 Orientation cage with tarp shield. (B) Orientation cage without tarp shield. (C) Dimensions.

536 Figure 3 Example of the radR interface. The image shows two frames from a video taken from

537 the bottom center of the cage. The perch exclusion zone is the area within the circular perch.

538 Figure 4 Average hybrid index and sex proportion was relatively constant over the migratory

539 period. (A) Average hybrid index over the fall migratory period. Hybrid index was based on

540 three nuclear genetic markers and was equivalent to the proportion of myrtle alleles (i.e. $0=$ all

541 Audubon's alleles; 1 = all myrtle alleles). (B) Proportion of daily sample that were male, as

542 determined by molecular sexing.

543 Figure 5 Activity of birds over the evening during the orientation trials. Each point is the

544 number of objects identified by radR over a 5-minute time period with the time relative to sunset. 
545 The grey points are the raw data from all individuals. The connected, filled circles are the

546 averaged points over a 20-minute window. Peak activity occurs approximately 20 minutes before

547 sunset.

548 Figure 6 Orientation of yellow-rumped warblers in relation to genetic ancestry. (A) Orientation

549 of all individuals in the study. (B-F) Orientation of individuals grouped according to genetic

550 hybrid index. 


\section{Figure 1}

Distribution and band recoveries of Audubon's and myrtle warblers, and location of study site.

(A) Banding data obtained from Brewer et al. (2006) and the Canadian Bird Banding Office (2013). Note that there is a distinct subspecies of Audubon's warbler, the black-fronted warbler, that occurs in Mexico and is not distinguished on this map. (B) Sites of capture for migratory yellow-rumped warblers with $(\mathrm{C})$ the site of the orientation assays. Map data: Google, DigitalGlobe.

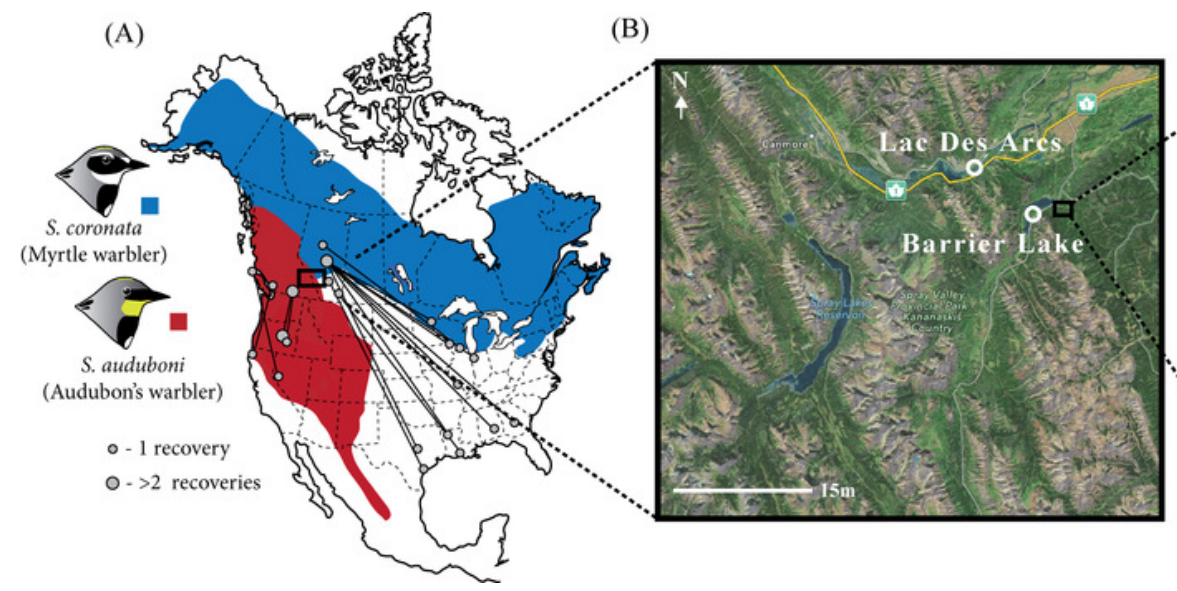

(C)

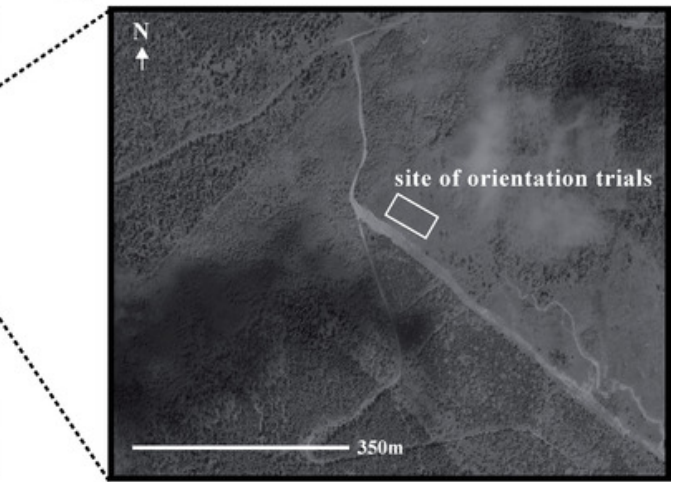


Figure 2

Holding and orientation cages as modified from Fitzgerald and Taylor (2008).

(A) Orientation cage with tarp shield. (B) Orientation cage without tarp shield. (C) Dimensions.

(A)

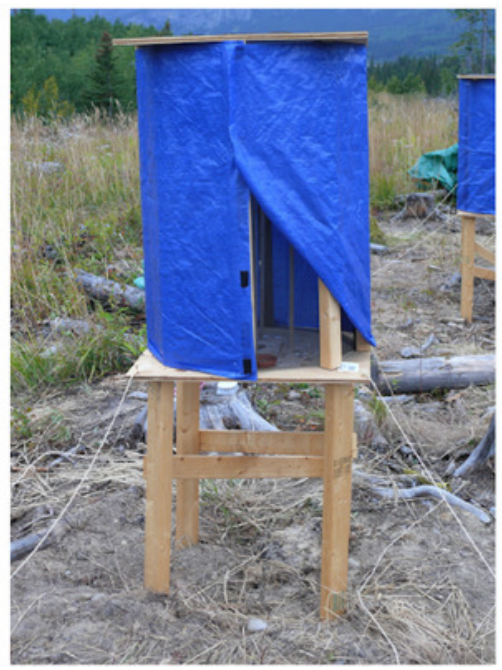

(B)

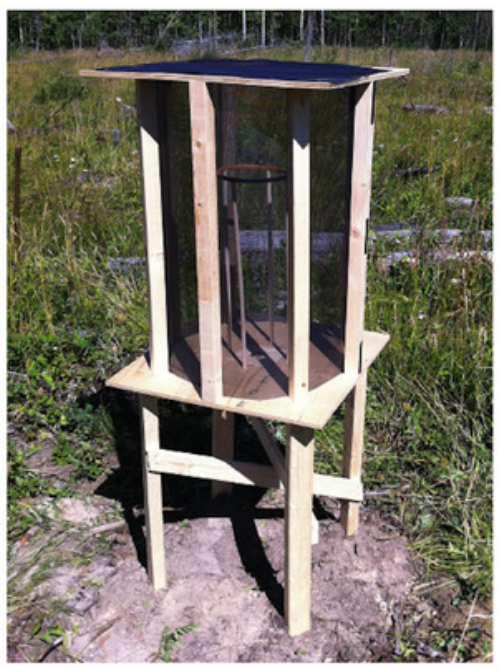

(C)

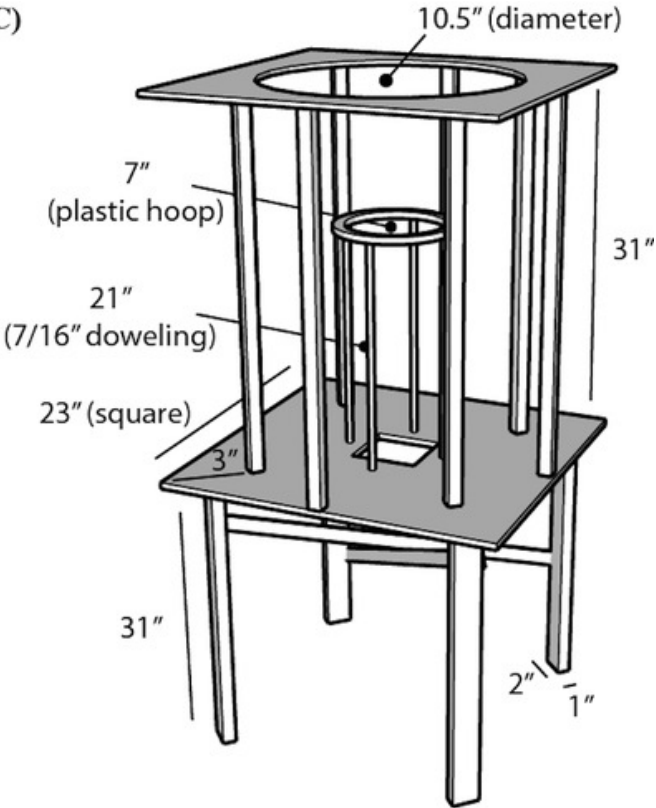




\section{Figure 3}

Example of the radR interface.

The image shows two frames from a video taken from the bottom center of the cage. The perch exclusion zone is the area within the circular perch.

\section{(A)}

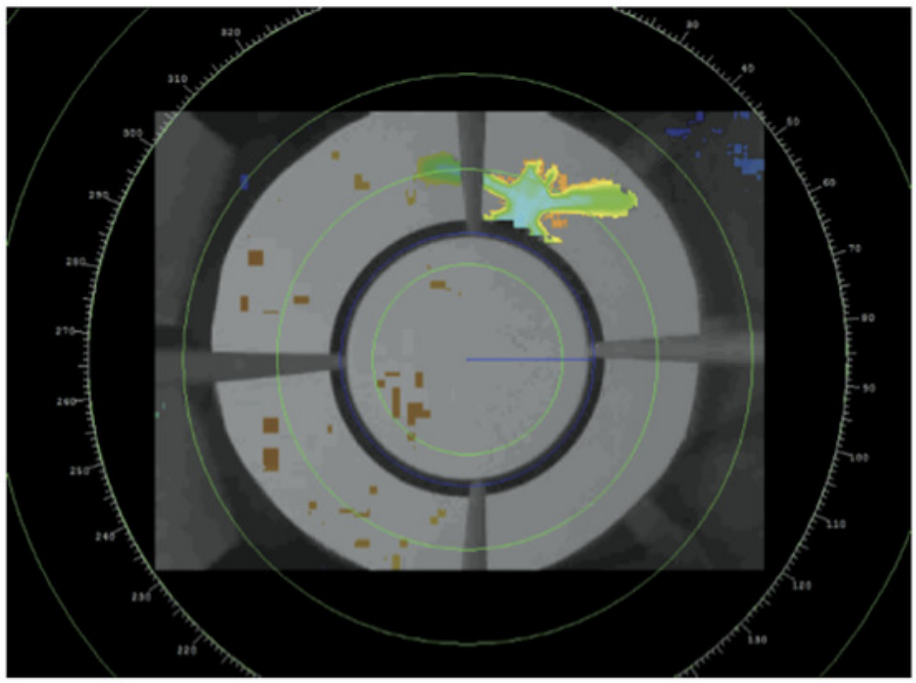

(B)

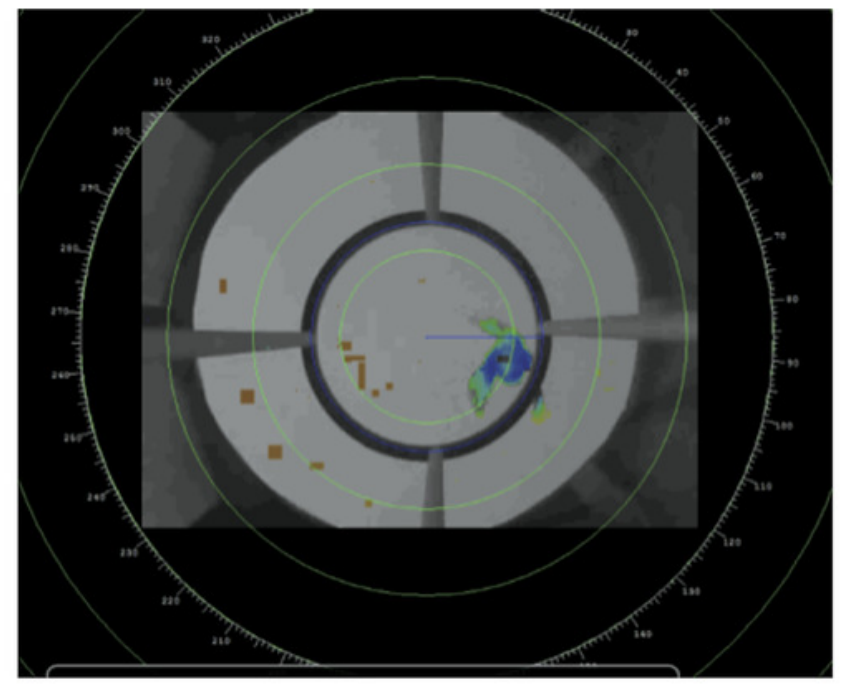


Figure 4

Average hybrid index and sex proportion is relatively constant over the migratory period.

(A) Average hybrid index over the fall migratory period. Hybrid index was based on three nuclear genetic markers and was equivalent to the proportion of myrtle alleles (i.e. $0=$ all Audubon's alleles; 1 = all myrtle alleles). (B) Proportion of daily sample that were male, as determined by molecular sexing.

(A)

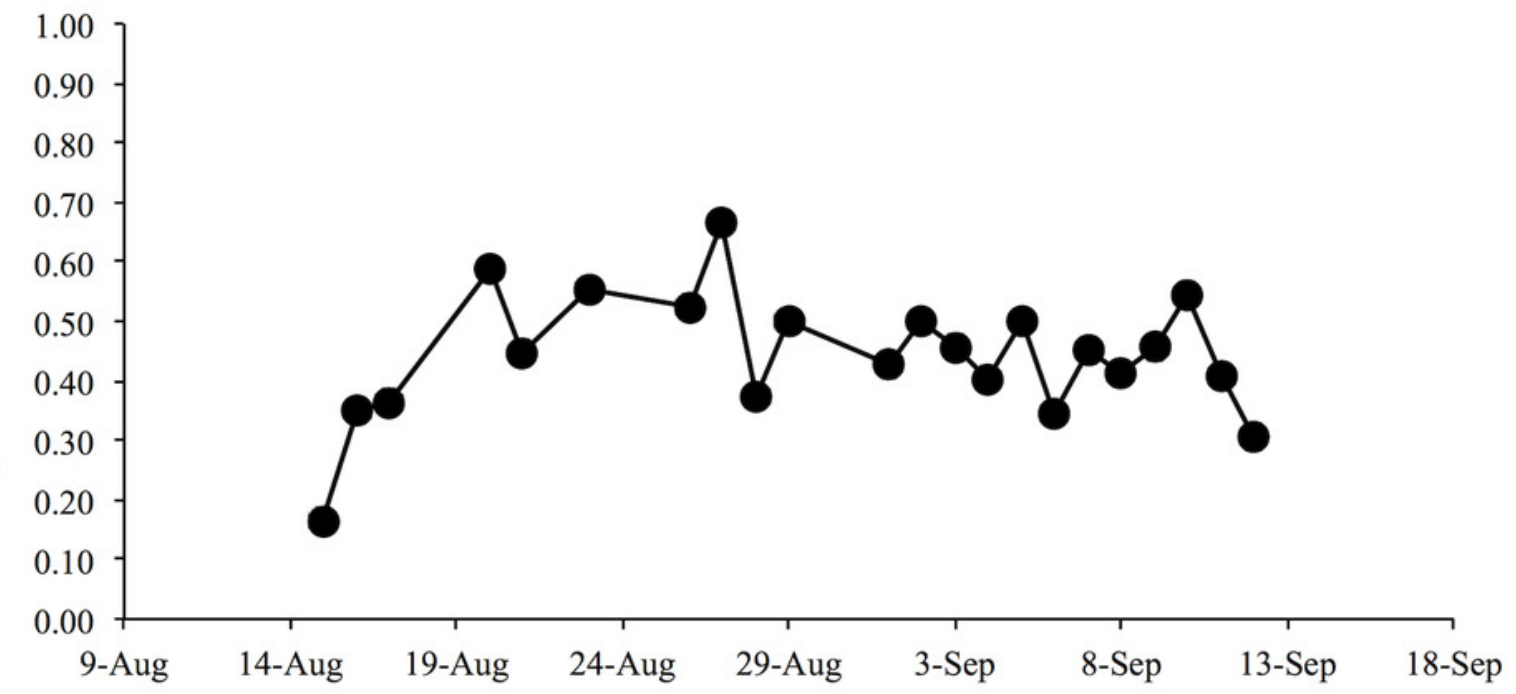

(B)

Allele

Frequency

(0 - all Audubon's

alleles,

1 - all myrtle alleles)

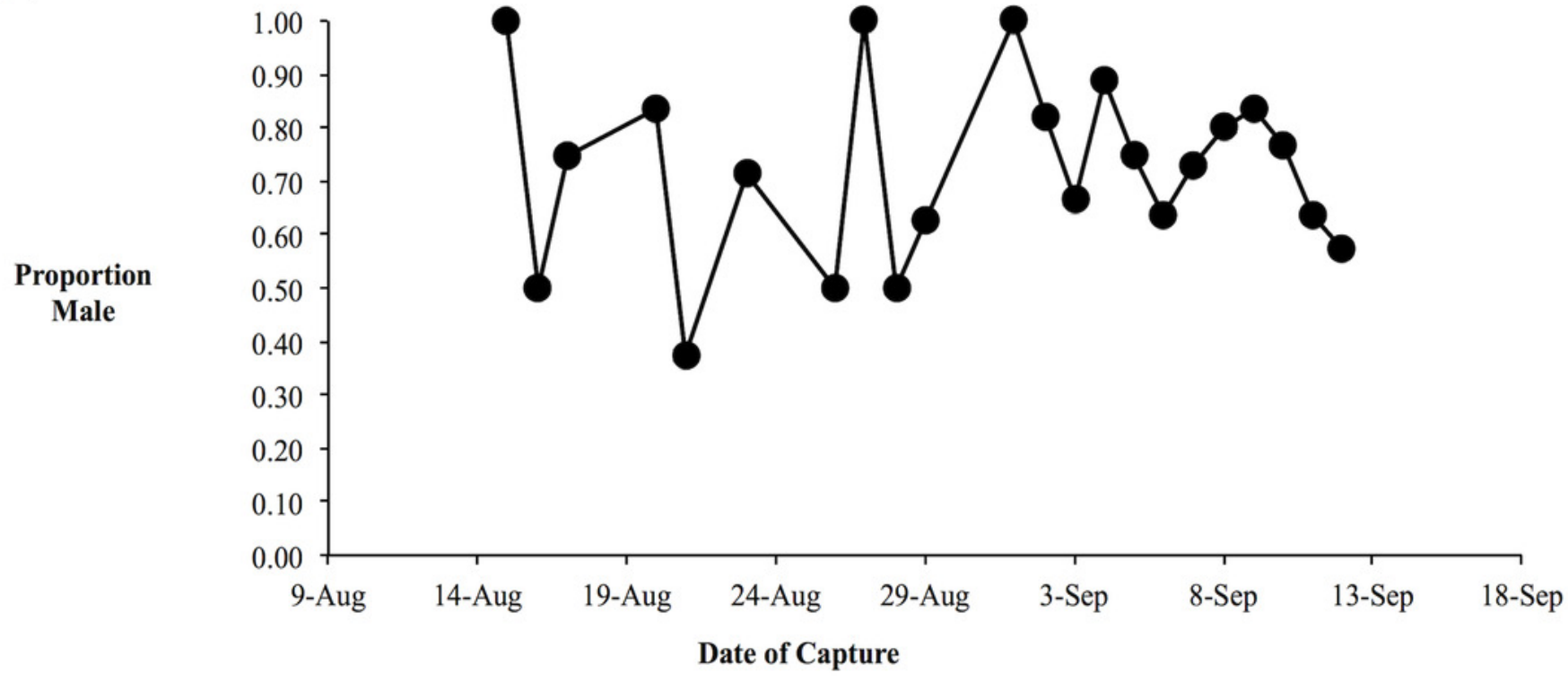




\section{Figure 5}

Activity of birds over the evenings during the the orientation trials.

Each point is the number of objects identified by radR over a 5 -minute time period with the time relative to sunset. The grey points are the raw data from all individuals. The connected, filled circles are the averaged points over a 20-minute window. Peak activity occurs approximately 20 minutes before sunset.

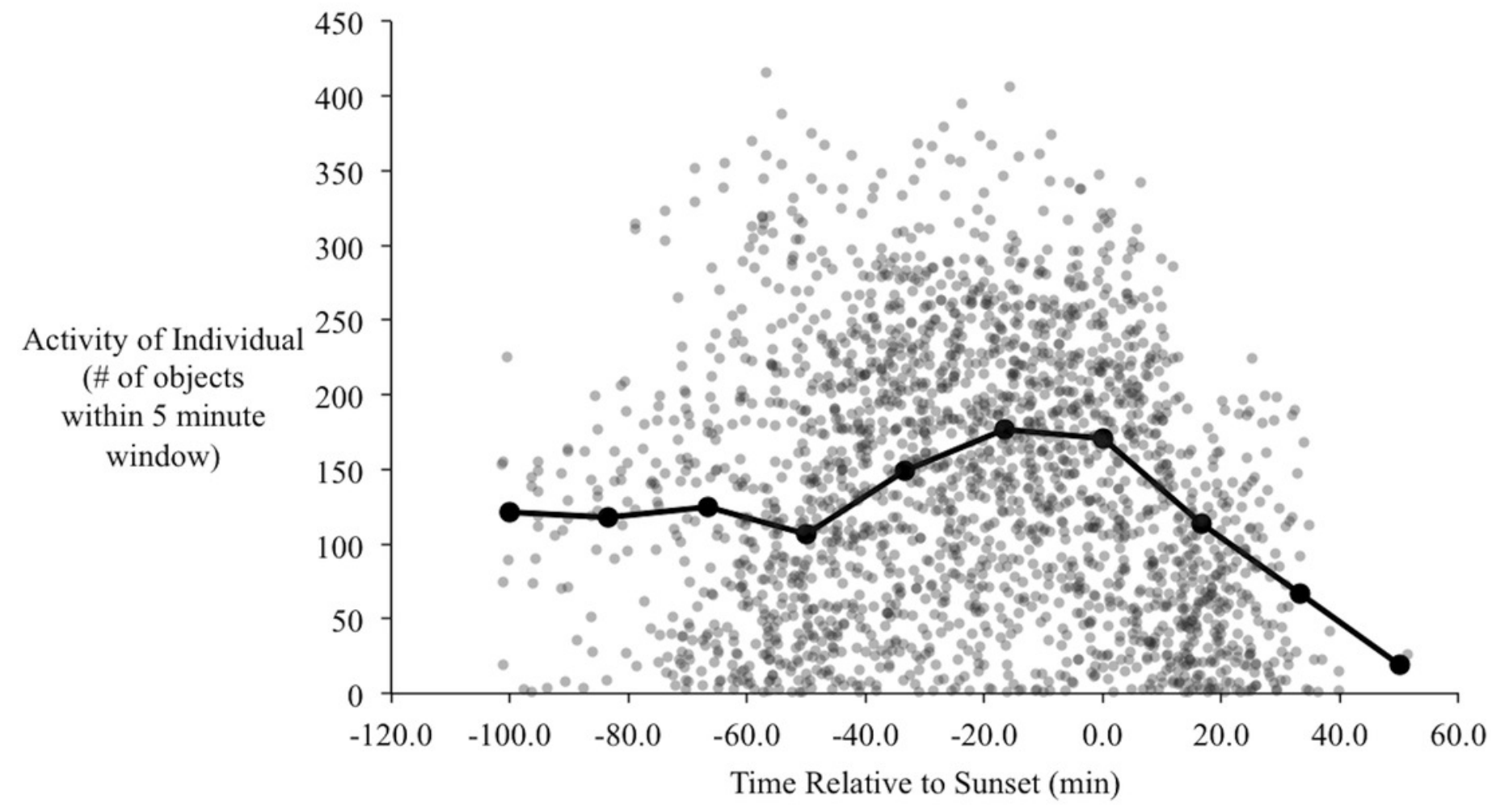




\section{Figure 6 (on next page)}

Orientation of yellow-rumped warblers in relation to genetic ancestry.

(A) Orientation of all individuals in the study. (B-F) Orientation of individuals grouped according to genetic hybrid index. 
(A)

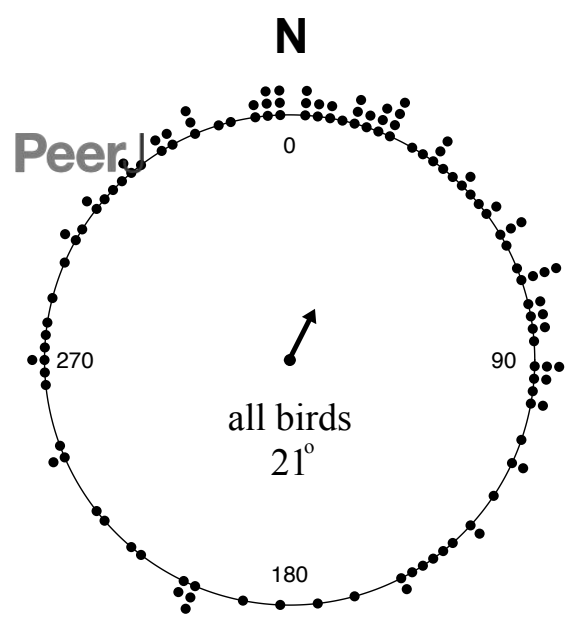

(C)

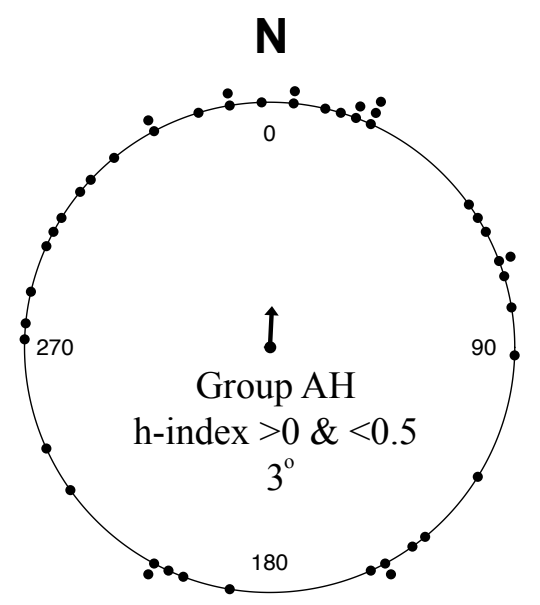

(E)

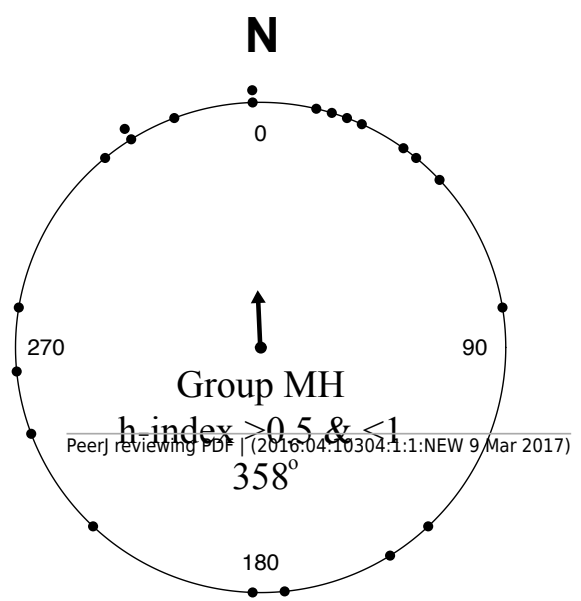

(B)

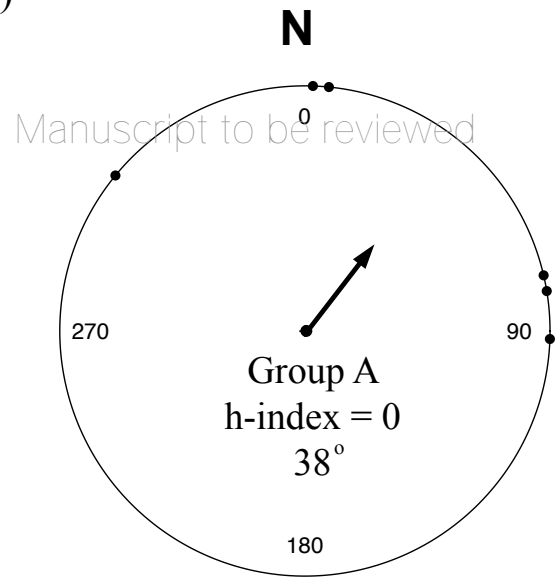

(D)

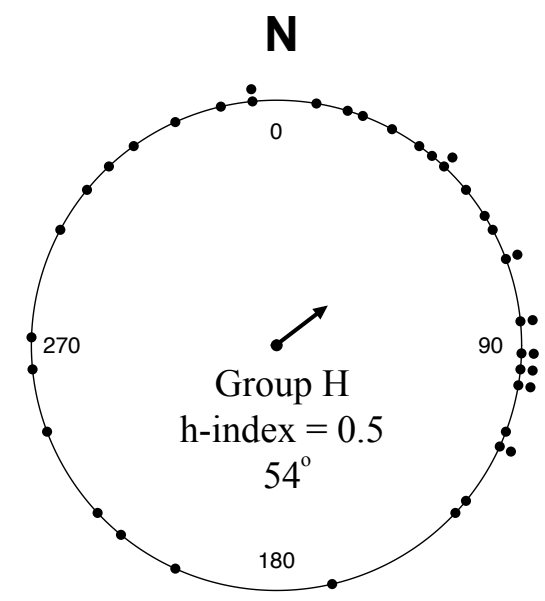

(F)

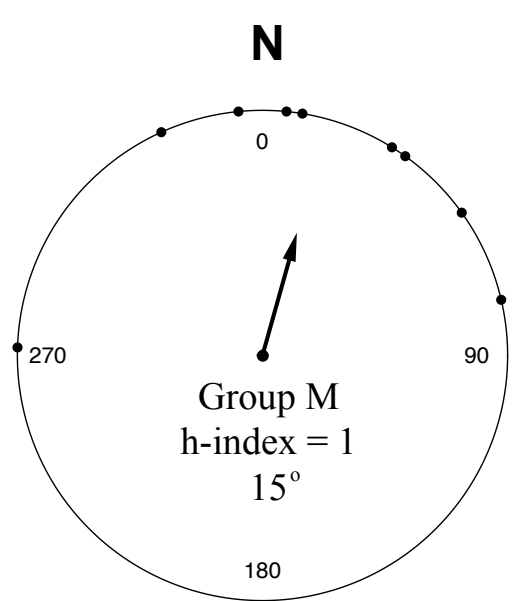

